\title{
Prediabetes en Perú: consenso de expertos
}

\section{Prediabetes in Peru: Consensus of experts}

\author{
Segundo Seclén-Santiesteban¹, Jaime Villena-Chávez², Miguel Pinto-Valdivia², José L. Paz-Ibarra², \\ Ray Ticse-Aguirre ${ }^{4}$, Jesús Rocca-Nación ${ }^{5}$, Harold Torres-Aparcana ${ }^{6}$, Juan C. Lizarzaburu-Robles ${ }^{7}$, \\ Alberto Teruya-Gibu ${ }^{8}$ y Rafael Bolaños-Díaz ${ }^{9 *}$ \\ ${ }^{1}$ Unidad de Diabetes, Hipertensión y Lípidos, Universidad Peruana Cayetano Heredia; ${ }^{2}$ Departamento de Endocrinología, Hospital Nacional \\ Cayetano Heredia; ${ }^{3}$ Departamento de Endocrinología, Hospital Nacional Edgardo Rebagliatti Martins; ${ }^{4}$ Departamento de Endocrinología, \\ Hospital Cayetano Heredia; ${ }^{5}$ Departamento de Endocrinología, Clínica Ricardo Palma; ${ }^{6}$ Departamento de Endocrinología, Clínica San Felipe; \\ ${ }^{7}$ Departamento de Endocrinología, Hospital Central de la Fuerza Aérea del Perú; ${ }^{8}$ Departamento de Endocrinología, Hospital Nacional Edgardo \\ Rebagliatti Martins; ${ }^{9}$ Organización Médica para el Desarrollo de la Salud, ODDS PERU. Lima, Perú
}

\section{RESUMEN}

Objetivo: Recabar información de prediabetes en el contexto peruano. Métodos: Se convocó a 9 expertos nacionales y se elaboró un cuestionario con puntuaciones para dimensiones de tamizaje, diagnóstico, herramientas para determinar riesgo de progresión a DM2, factores de riesgo y manejo de prediabetes para la prevención de la DM2. El consenso se realizó en 3 rondas, siendo la última, presencial. Hallazgos: La glucosa plasmática en ayunas es considerada la mejor prueba para el tamizaje de prediabetes. El mejor punto de corte es el recomendado por la ADA $(100 \mathrm{mg} / \mathrm{dl}$ a $125 \mathrm{mg} / \mathrm{dl}$ o 5.6-6.9 mmol/L). Todo paciente con glucosa plasmática en ayunas alterada debe ser sometido a una prueba de tolerancia oral a la glucosa con $75 \mathrm{~g}$ de glucosa anhidra. El estudio PERUDIAB se considera el mejor estimador poblacional de glucosa alterada en ayuno con una prevalencia de $22.4 \%$. Las opciones son: cambio de estilo de vida o éste acompañado de metformina. Conclusión: Este documento es clave para establecer políticas de prevención de prediabetes llegando a identificar poblaciones de riesgo y herramientas para el tamizaje.

Palabras clave: Prediabetes. Hiperglucemia. Metformina.

\section{ABSTRACT}

Objective: To collect information of prediabetes in the Peruvian context. Methods: 9 national experts were summoned and a scoring questionnaire was prepared for screening dimensions as diagnosis, tools to determine the risk of progression to $\mathrm{DM}_{2}$, risk factors and management of prediabetes for the prevention of DM2. The consensus was made in three rounds, being the third one, face-to-face. Findings: Fasting plasma glucose is the best test for prediabetes screening. The best cut-off point is the one recommended by the American Diabetes Association ( $100 \mathrm{mg} / \mathrm{dl}$ to $125 \mathrm{mg} / \mathrm{dl}$ or 5.6-6.9 mmol/L). Every patient with altered fasting plasma glucose should undergo an oral glucose tolerance test with $75 \mathrm{~g}$ of anhydrous glucose. PERUDIAB study is the best population analysis that estimated an impaired fasting glucose prevalence of $22.4 \%$. The options are: lifestyle change or this one but with metformin. Conclusion: This document is essential to establish pre-diabetes prevention policies, identifying risk populations and screening tools.

Key words: Prediabetes. Hyperglycemia. Metformin.
*Rafael Bolaños-Díaz

Fecha de recepción: 11-11-2019

Fecha de aceptación: 10-02-2020

E-mail: rbolanosdaoddsperu.com; rbolanosdayahoo.es

DOI: 10.24875/ALAD.20000409
Disponible en internet: 26-03-2020 Rev ALAD. 2020;10:66-79

2248-6518 / @ 2020 Asociación Latinoamericana de Diabetes. Publicado por Permanyer. Este es un artículo open access bajo la licencia CC BY-NC-ND (http://creativecommons.org/licenses/by-nc-nd/4.0/). 


\section{INTRODUCCIÓN}

La prevalencia e incidencia de diabetes mellitus tipo 2 (DM2) está en aumento y se estima que aproximadamente 425 millones de adultos (20-79 años) viven con esta condición, con un impacto mayor en países de bajos a medianos ingresos ${ }^{1,2}$. En el Perú, basado en estudios poblacionales se pudo estimar que la prevalencia de diabetes se encuentra en un rango desde un 5.1\%, según el estudio del Instituto Nacional de Salud del Perú realizado entre 2004 y 2005 en sujetos mayores de 35 años, hasta un $7 \%$, de acuerdo con el estudio PERUDIAB realizado entre 2010 y 2012 y que incluyó sujetos mayores de 25 años $^{3}$. Se ha demostrado que el impacto de diabetes en el Perú es significativo, representa la octava causa de muerte, la sexta causa de ceguera y la primera causa de enfermedad renal crónica y necesidad de diálisis en el país ${ }^{4}$. Además, afecta al $31.5 \%$ de personas con infarto agudo de miocardio, representa del 10 al 15\% de los accidentes cerebrovasculares y es la primera causa de amputación no traumática ${ }^{4}$. Además, en el Perú se estima una incidencia de diabetes de 19.5 nuevos casos por 1,000 personas-años (13.9-28.3)

El diagnóstico temprano, así como la detección de factores de riesgo para prevenir el desarrollo de DM2 son fundamentales para evitar el aumento de esta enfermedad. La prediabetes es un estado metabólico intermedio entre el estado de glucemia normal y la $\mathrm{DM} 2^{6}$. Se estima que anualmente entre el 5 y el 10\% de personas en estado de prediabetes progresarán a DM2 y de la misma manera este porcentaje tendría el potencial de revertir este estado y volver a la normoglucemia ${ }^{6}$. Es por esto que la identificación de personas con prediabetes es fundamental para la prevención de la DM2. Entre las intervenciones más estudiadas se encuentra la modificación del estilo de vida, así como medidas farmacológicas ${ }^{6}$. A pesar de la importancia de identificar prediabetes y factores de riesgo para detener la epidemia de DM2, se dispone de poca información local con respecto a la epidemiología, manejo y factores de riesgo de prediabetes en el Perú.

Ante un escenario de incertidumbre, en el cual no se tiene literatura disponible acerca de datos específicos, se pueden realizar metodologías de consenso de expertos?.

Por lo tanto, ante la escasez de información publicada con respecto a prediabetes, este trabajo tiene como objetivo recabar la información respecto a aspectos relevantes de prediabetes y proponer acciones en el contexto peruano con base en un ejercicio de consenso de expertos nacionales en el manejo de prediabetes.

\section{MATERIAL Y MÉTODOS}

Se convocó a nueve expertos en el manejo de pacientes con prediabetes en el Perú. Estos fueron seleccionados de acuerdo con su participación en sociedades de manejo de diabetes nacionales e internacionales. Las filiaciones y cargos relevantes de los expertos seleccionados se presentan en el anexo 1. Se realizó un cuestionario que fue elaborado por un experto local en diabetes y epidemiología. Este cuestionario se presentó a los expertos seleccionados que fueron aclarando y modificando las preguntas cuando fuere necesario. El cuestionario final se encuentra en el anexo 2. Cada uno de los expertos recibió este cuestionario, que consistía en 30 preguntas que abarcaban dimensiones de tamizaje y diagnóstico, epidemiología, población en riesgo e intervenciones de manejo y prevención de prediabetes. Se realizó un Ilenado independiente de este cuestionario (primera ronda), en donde se 
aclararon y se tomaron en consideración nuevas preguntas o criterios adicionales. Luego de las modificaciones respectivas se volvió a repartir el cuestionario para que fuese completado independientemente por cada uno de los expertos (segunda ronda). Después del análisis de la segunda ronda de respuestas se realizó la sesión presencial, en la cual se resolvieron los aspectos en los que no existía consenso si fue posible. De la misma forma, se presentaron los resultados de consensos para que fueran validados por todos los expertos participantes.

La reunión presencial se desarrolló el día 1 de octubre del año 2019. En esta sesión se realizaron discusiones abiertas de las dimensiones del cuestionario y de desacuerdos.

Toda la información recabada fue analizada y compilada en secciones relevantes siguiendo el modelo de otros consensos previos descritos en la región ${ }^{8,9}$.

\section{Puntuación}

Todas las preguntas del cuestionario (Anexo 2) tuvieron que ser puntuadas en una escala del 1 al 9 , en donde se indicó marcar 1 si está «totalmente en desacuerdo» y 9 si está "totalmente de acuerdo» con afirmaciones presentadas. El valor 5 indicaba «ni de acuerdo ni en desacuerdo». Adicionalmente, puede distinguirse una valoración colorimétrica de las respuestas en tres regiones: desacuerdo (rojo), indiferente (amarillo) y de acuerdo (verde) (Fig. 1).

Esta puntuación de nueve niveles para el análisis de nueve expertos se basó en la metodología RAND/ $\mathrm{UCLA}^{10,11}$. Para analizar si había o no consenso ejecutamos las siguientes reglas de puntuación:

- En cada indicación, existe "acuerdo» (para un panel de nueve expertos) cuando no más de dos

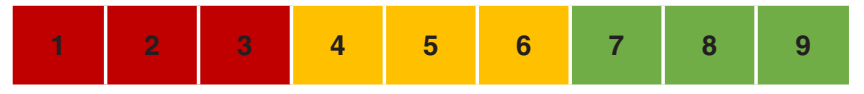

Figura 1. Escala de puntuación y niveles colorimétricos de respuesta.

panelistas la han puntuado fuera de la región de tres puntos (1-3, 4-6, 7-9) que contiene a la mediana de las nueve valoraciones.

- Existe «desacuerdo» cuando las puntuaciones de tres o más panelistas están en la región de 1-3, y tres o más en la región 7-9.

- Las indicaciones en las que no existe «acuerdo» ni «desacuerdo» son consideradas como «indeterminadas».

- Todas las indicaciones puntuadas como «desacuerdo» 0 «indeterminadas» pasan a la siguiente ronda.

\section{RESULTADOS}

\section{Diagnóstico de prediabetes y tamizaje en población general peruana}

Existen diferentes definiciones para el diagnóstico de prediabetes que varían con respecto a las herramientas diagnósticas utilizadas y en los puntos de corte. Se consideró que las definiciones más relevantes fueron las descritas por la Asociación Americana de Diabetes (ADA $)^{12}$ y la Organización Mundial de la Salud (OMS)13 (Tabla 1).

Los expertos coincidieron en la importancia de resaltar el uso diferencial de pruebas diagnósticas dependiendo de si se utilizan con el objetivo de realizar un diagnóstico o un tamizaje. Se obtuvo 
TABLA 1. Definiciones de la Asociación Americana de Diabetes (ADA) y la Organización Mundial de la Salud (OMS) para prediabetes

\begin{tabular}{|l|c|c|c|}
\hline Fuente/test & Hemoglobina glucosilada & Glucosa plasmática en ayunas & $\begin{array}{c}\text { Glucosa } 2 \text { horas post test } \\
\text { de tolerancia oral a la glucosa }\end{array}$ \\
\hline ADA, 2017 & $\begin{array}{c}5.7-6.4 \% \\
(39-46 \mathrm{mmol} / \mathrm{mol})\end{array}$ & $\begin{array}{c}100-125 \mathrm{mg} / \mathrm{dl} \\
(5.6-6.9 \mathrm{mmol} / \mathrm{l})\end{array}$ & $\begin{array}{c}140-199 \mathrm{mg} / \mathrm{dl} \\
(7.8-11.0 \mathrm{mmol} / \mathrm{l})\end{array}$ \\
\hline OMS, $2001^{13}$ & No definido & $\begin{array}{c}110-125 \mathrm{mg} / \mathrm{dl} \\
(6.1-6.9 \mathrm{mmol} / \mathrm{l})\end{array}$ & $\begin{array}{c}140-199 \mathrm{mg} / \mathrm{dl} \\
(7.8-11.0 \mathrm{mmol} / \mathrm{l})\end{array}$ \\
\hline
\end{tabular}

consenso en reconocer las dos definiciones como aceptables en el diagnóstico de prediabetes. Si bien la definición de la ADA toma en cuenta valores de hemoglobina glucosilada dentro de sus criterios diagnósticos, las definiciones de la ADA y la OMS tienen los mismos puntos de corte con respecto a la glucemia de $2 \mathrm{~h}$ poscarga de glucosa. La mayor diferencia entre las dos definiciones se encuentra en los puntos de corte para la definición de prediabetes utilizando la glucosa plasmática en ayunas.

Se discutió que, para el contexto peruano, los puntos de corte de la ADA utilizando glucosa plasmática en ayunas serían más adecuados que los criterios de la OMS, dado que tendría implicación en el tamizaje. De esta forma, tener un punto de corte más bajo podría aumentar la sensibilidad de la prueba diagnóstica y ayudarnos a identificar más casos en la población general. Así mismo se estableció que, siempre que sea posible, después de encontrar un valor alterado de glucosa plasmática en ayunas se debe confirmar el diagnóstico de prediabetes por medio de la prueba de tolerancia oral a la glucosa con 75 g de glucosa anhidra de carga. No se llegó a un consenso respecto al uso de hemoglobina glucosilada para el tamizaje o para confirmar el diagnóstico de prediabetes debido a la limitada disponibilidad y estandarización de esta prueba en nuestro país.
Respecto al tamizaje en población general, los expertos consensuaron en que se debería realizar en población mayor de 35 años. Si bien se discutieron diversos puntos de corte de edad (como en mayores de 40 o de 45 años), finalmente se decidió tomar un punto de corte edad basado en la estratificación de edad utilizada en el estudio PERUDIAB5 ${ }^{5}$ Es importante recalcar que el estudio PERUDIAB incluyó población mayor de 25 años, pero evidenció que a partir de los 35 años la prevalencia de glucosa alterada en ayuno era mayor al $25 \%$, siendo mayor que en todos los otros grupos de edad analizados ${ }^{5}$.

\section{Evaluación de riesgo para prediabetes}

El cuestionario FINDRISC (Finnish Diabetes Risk Score) es una herramienta para la identificación de pacientes con prediabetes que ha demostrado ser económica, rápida y fiable ${ }^{14}$. Esta herramienta se ha validado en diferentes países, incluyendo Colombia ${ }^{15}$ y Venezuela ${ }^{16}$, para la identificación de pacientes con prediabetes con resultados positivos.

Si bien hubo consenso de los expertos en que la herramienta FINDRISC puede ser utilizada en el contexto peruano, mencionaron que esta utilización debe ser hecha después de una validación local tanto cualitativa como cuantitativa. Es así que se discutieron los criterios o parámetros importantes que se deberían tomar en cuenta si es que se 
TABLA 2. Criterios que considerar en la validación local de la herramienta FINDRISC (Finnish Diabetes Risk Scorel

\begin{tabular}{|l|l|}
\hline \multirow{7}{*}{ Consenso } & Criterios \\
\hline & $\begin{array}{l}\text { Validación local de la puntuación de riesgo para } \\
\text { diabetes de la herramienta }\end{array}$ \\
\cline { 2 - 3 } $\begin{array}{c}\text { Validación de adecuados puntos de corte del } \\
\text { perímetro abdominal en la evaluación de } \\
\text { riesgo para el contexto de población peruana }\end{array}$ \\
\hline \multirow{2}{*}{$\begin{array}{c}\text { Reformular la pregunta sobre si come frutas y } \\
\text { verduras de acuerdo a un contexto local. Por } \\
\text { ejemplo, considerar llamarlo frutas, vegetales } \\
\text { y hortalizas }\end{array}$} \\
\cline { 2 - 2 } & $\begin{array}{c}\text { Adicional a la pregunta de medicación de la } \\
\text { hipertensión arterial, agregar si el individuo } \\
\text { toma medicamentos para la dislipidemia }\end{array}$ \\
\hline
\end{tabular}

hiciera una validación nacional de la herramienta. La lista de estos criterios y los grados de consenso son presentados en la tabla 2.

\section{Epidemiología de la prediabetes}

Existen escasos estudios acerca de la prevalencia de prediabetes en el Perú. Sin embargo, se considera que el estudio más representativo es el PERUDIAB, el cual muestra que un $22.4 \%$ de la población peruana presenta glucosa alterada en ayuno ${ }^{5}$. Hubo un acuerdo en que este valor podría estar subestimado y que una estimación más exacta se lograría haciendo un estudio nacional con test de tolerancia oral a la glucosa.

\section{Factores de riesgo para desarrollar prediabetes}

Si bien existen criterios específicos considerados en herramientas como el FINDRISC para valorar el riesgo, se intentó identificar población específica de mayor riesgo en un contexto local. La lista de las
TABLA 3. Población peruana en mayor riesgo de prediabetes

\begin{tabular}{|l|l|}
\hline & Población en riesgo \\
\hline Consenso & - Mayores de 35 años \\
& - Obesos \\
& - Población de la costa \\
& - Migrantes de zonas rurales a zonas urbanas \\
& - Antecedentes de diabetes gestacional \\
& - Antecedente de parto de bebe macrosómico \\
& (hijo de 4,000 g o más) \\
& - Individuos con acantosis nigricans \\
& - Mujeres con síndrome de ovario poliquístico \\
& - Individuos con síndrome metabólico \\
& - Individuos con hipertensión arterial \\
& - Individuos con hipertrigliceridemia \\
& - Individuos con familiares de primer grado \\
& con DM2 \\
\hline
\end{tabular}

DM2: diabetes mellitus tipo 2.

poblaciones de riesgo que se consensuaron se presenta en la tabla 3.

Aunque se discutió acerca de considerar al sexo masculino como una población de alto riesgo, no hubo un consenso entre los expertos con respecto a este criterio. Además, se tomó en consideración a la población que consume alimentos ultraprocesados, concluyéndose que era muy difícil identificar a esta población específicamente, por lo que no hubo consenso en su inclusión en la población en riesgo.

\section{Intervenciones para la prevención y manejo de prediabetes poblacional}

Los expertos reconocieron que el tamizaje y la evaluación integral de personas de riesgo es fundamental para la prevención de prediabetes, ya que nos llevaría a hacer una identificación temprana.

El fomentar un cambio en el estilo de vida se consideró una intervención efectiva en cuanto a costo para la prevención de prediabetes. Se mencionó 
que las intervenciones de prevención educadoras para mejorar el estilo de vida de las personas deben darse por medio de comunicación masiva e incluyendo técnicas modernas de comunicación como son las redes sociales a nivel poblacional e individual, por lo cual se debe contar con el apoyo de las autoridades.

Si bien se han observado beneficios en otros países con intervenciones educativas complejas e intensivas como el estudio US-DPP (United States-Diabetes Prevention Program) o Prevent-DM ${ }^{14,17}$, los expertos llegaron a un acuerdo en mencionar que ese tipo de intervenciones solo sería aplicable si se asegura la sostenibilidad de un programa con esa complejidad. Dado que estas intervenciones requieren un seguimiento y medición continua de las metas, así como la inversión en recursos humanos especializados, los expertos consideran que intervenciones de este tipo no serían aplicables actualmente en nuestro contexto.

Sin embargo, es importante recalcar que esto no quiere decir que este tipo de intervenciones no sean efectivas, sino que debe asegurarse su sostenibilidad en el tiempo, incluso con los recursos actualmente limitados e invertidos en programas de prevención de diabetes en el Perú. Además, en el contexto peruano no se consideraron como medidas efectivas respecto al costo el impuesto a las bebidas azucaradas ni las políticas agropecuarias.

Con respecto a las intervenciones farmacológicas, se consideró como medida terapéutica el uso de metformina en nuestra población, dado que ha mostrado ser beneficiosa en prediabetes, y que una dosis de $850 \mathrm{mg}$ dos veces al día reduce la progresión de prediabetes a diabetes en un 31\% comparado con placebo ${ }^{14,18}$. Es importante recalcar que se debe evaluar si existen o no contraindicaciones para el inicio de metformina en todos los pacientes.
Los expertos alcanzaron el consenso en que la terapia a indicar en pacientes con prediabetes dependía siempre del caso clínico específico y podía variar dependiendo de los factores de riesgo de los pacientes, sobre todo en el contexto de un síndrome metabólico. Por ello es importante realizar una valoración de factores de riesgo integral antes de indicar una terapia de manejo en cada paciente. Entonces se puede indicar cambio de estilo de vida solamente o cambio de estilo de vida concomitantemente a la indicación de metformina. Se han descrito recomendaciones acerca de estas intervenciones en otros consensos publicados ${ }^{19}$.

En el caso de indicarse un cambio de estilo de vida, este debe ser reevaluado cada tres meses. Los parámetros para tomar en cuenta son: la evaluación integral del paciente (incluyendo peso, índice de masa corporal [IMC] y perímetro abdominal) y la glucosa plasmática en ayunas ${ }^{19}$.

En caso de indicar manejo con metformina (liberación inmediata) se debe prescribir una dosis de $850 \mathrm{mg}$ por día después del almuerzo durante un mes. Luego de este mes se evalúa la tolerancia al fármaco. Si la tolerancia es buena, se continúa el tratamiento con metformina $850 \mathrm{mg}$ dos veces al día. Cada tres meses se debe realizar un monitoreo del paciente mediante un examen integral (incluyendo peso, IMC y perímetro abdominal), así como de glucosa plasmática en ayunas. La decisión de continuar, modificar o descontinuar la terapia se decide después de un año de tratamiento. Después de un año, se indica al paciente descontinuar el fármaco durante dos semanas para realizar un test de tolerancia oral de glucosa; con esta información se reevaluará continuar o cambiar el tratamiento. Existe otro tipo de metformina que es de liberación prolongada y se considera sobre todo para los casos de intolerancia a metformina de acción rápida. En este caso la dosis es de 750 mg dos veces al día ${ }^{19}$. 


\section{DISCUSIÓN}

Este es el primer consenso de expertos realizado exclusivamente para establecer el diagnóstico, prevención y tratamiento de prediabetes en el Perú. Es importante mencionar que los consensos de expertos se utilizan cuando la información en la literatura es escasa y no se pueden realizar búsquedas sistemáticas para contestar determinadas preguntas acerca de un tema. Si bien la riqueza de esta metodología es poder llegar a la mejor estimación posible con los recursos disponibles, su mayor limitación es la representatividad de estas opiniones de experto. Es por esto por lo que intentamos invitar a doctores que hayan tenido algún cargo que les permita tener una visión nacional del problema de diabetes. Las características de los profesionales incluidos se encuentran en el anexo 1.

De la misma forma, se tiene que mencionar que una de las limitaciones más importantes es inherente a la metodología, en la cual no podemos probar lo dicho o recomendado basándonos en publicaciones científicas sino en lo discutido en el consenso. En esta oportunidad utilizamos una herramienta que nos ayudó a identificar los consensos de una manera más equitativa de acuerdo al número de expertos incluidos ${ }^{10,11}$.

La mayoría de las recomendaciones que se consignan en este consenso se encuentran dentro de los lineamientos de anteriores consensos en Latinoamérica ${ }^{8,20}$. Si bien se hacen recomendaciones con respecto a las pruebas diagnósticas o cuestionarios de preferencia en población peruana, es importante tomar en cuenta que la preferencia de una tecnología sobre otra tiene que ser evaluada de acuerdo con la coyuntura del país y si fuera posible basándose en análisis de costo-beneficio.
Las ventajas de considerar un punto de corte más inclusivo (ADA: 100-125 mg/dl o 5.6-6.9 mmol/l) utilizando la glucosa plasmática en ayunas son que se puede llegar a una mejor sensibilidad tomando en cuenta más pacientes con una prueba poco costosa en Perú. Además, todos los centros de salud tienen disponibilidad de glucosa plasmática, mientras que esto no sucede en el caso de la hemoglobina glucosilada. Es importante tomar en cuenta las diferencias en las definiciones de prediabetes y cómo estas pueden modificar los estimadores poblacionales. Por ejemplo, recientemente se publicó un estudio realizado en tres localidades de Perú en el cual se evalúa la prevalencia de prediabetes utilizando diferentes definiciones. En dicho estudio se encontró que, al analizar los individuos incluidos bajo la definición de la OMS (tomando en cuenta solo la glucosa plasmática en ayunas entre 110 y 125 mg/dl) la prevalencia es del $6.5 \%$ (intervalo de confianza [IC] 95\%: 5.6-7.6\%) mientras que si se utiliza los criterios diagnósticos de la ADA (glucosa plasmática en ayunas entre 100 y $125 \mathrm{mg} / \mathrm{dl}$ o hemoglobina glucosilada entre el 5.7 y el $6.4 \%$ ) se encuentra una prevalencia del 53.6\% (IC 95\%: 51.6-55.6\%) ${ }^{21}$. De esta forma se comprueba la importancia que tiene estandarizar estas definiciones y considerarlas en futuras guías de práctica clínica y herramientas para el monitoreo de intervenciones de salud.

La utilización de un cuestionario como el FINDRISC sería ideal en nuestro país pero, como ya fue antes mencionado, es fundamental realizar una validación cuantitativa y cualitativa. Con respecto a los factores de riesgo, muchos de estos coinciden con los factores de riesgos ya descritos en la literatu$\mathrm{ra}^{20}$. Sin embargo, existen otros factores de riesgo que se han encontrado solo en población peruana. En el estudio PERUDIAB, uno de los pocos estudios de representatividad nacional en el Perú, se puede observar que los pobladores de la costa tienen mayor riesgo de prediabetes en comparación con la población de la sierra5. 
Teniendo en cuenta que el aumento en la prevalencia de obesidad es evidente en Sudamérica, y sumado a otros factores de riesgo de la población, se espera un incremento en la incidencia y prevalencia de prediabetes y diabetes en la región ${ }^{22}$. Es por esto por lo que es fundamental compilar la mejor evidencia posible acerca de la prediabetes y de esta forma tomar esta información como un recurso para producir políticas factibles y focalizadas en las necesidades de la población peruana. Si bien se insta a realizar investigación primaria acerca del diagnóstico, la prevención y el manejo de la prediabetes, este documento proporciona una aproximación a estos puntos.

\section{CONCLUSIONES Y RECOMENDACIONES}

A continuación presentamos las conclusiones de este consenso:

- La glucosa plasmática en ayunas se considera la mejor prueba para el tamizaje de prediabetes en la población peruana. Se tiene consenso en que el mejor punto de corte para esta prueba (en este escenario) es el recomendado por la ADA (100-125 mg/dl o 5.6-6.9 mmol/l).

- Para el contexto peruano, actualmente no se considera la hemoglobina glucosilada ni la combinación de glucosa plasmática en ayunas y hemoglobina glucosilada como pruebas de tamizaje para prediabetes.

- Todo paciente con glucosa plasmática alterada debería someterse a una prueba de tolerancia a la glucosa (140-199 mg/dl o 7.8-11.0 mmol/l) para definir la presencia de tolerancia alterada a la glucosa y definir prediabetes.
- El tamizaje se debería realizar en mayores de 35 años.

- La herramienta de valoración de riesgo FINDRISC puede utilizarse en población peruana siempre y cuando sea validada para el contexto local.

- Se considera el estudio PERUDIAB como el mejor estimador poblacional de glucosa alterada en ayuno, con una prevalencia del $22.4 \%$.

- Se identificaron poblaciones específicas consideradas de riesgo, entre las que se mencionaron a individuos mayores de 35 años, obesos (incluyendo adolescentes obesos), población de la costa y migrantes de zonas rurales a zonas urbanas, entre otras.

- El tamizaje y evaluación integral de personas de riesgo es fundamental para la prevención de prediabetes, así como para su manejo.

- Fomentar un cambio en el estilo de vida se considera una intervención costo-efectiva para la prevención de la prediabetes. Sin embargo, se menciona que intervenciones educadoras intensivas para el cambio de estilo de vida poblacional, si bien han demostrado ser efectivas, requieren de un soporte financiero importante para asegurar la sostenibilidad en el sistema. Los expertos coinciden en que, actualmente, en el Perú se debe planificar un programa de ese tipo para que sea aplicable a nuestra realidad.

- Además, en el contexto peruano no se consideran medidas costo-efectivas el impuesto a las bebidas azucaradas ni las políticas agropecuarias.

- Los expertos alcanzaron el consenso en que la terapia que indicar en pacientes con prediabetes 
dependía siempre del caso clínico específico y podía variar dependiendo de los factores de riesgo y los criterios diagnósticos que presenten los pacientes, sobre todo en el contexto de un síndrome metabólico. Las dos opciones mencionadas para el manejo de prediabetes fueron: cambio de estilo de vida solamente o cambio de estilo de vida concomitantemente a la indicación de metformina. realizado experimentos en seres humanos ni en animales.

Confidencialidad de los datos. Los autores declaran que en este artículo no aparecen datos de pacientes.

Derecho a la privacidad y consentimiento informado. Los autores declaran que en este artículo no aparecen datos de pacientes.

\section{BIBLIOGRAFÍA}

Organización Médica para el Desarrollo de la Salud - ODDS PERU (consultora privada de investigación biomédica, www.oddsperu.com) ha financiado la logística para el desarrollo del consenso, sin tener injerencia alguna en los debates o en el contenido del manuscrito.

\section{CONFLICTO DE INTERESES}

J.L.P.I. ha sido advisory board y speaker para Merck Serono y Abbott Nutrition en los últimos dos años.

R.B.D. pertenece a ODDS PERU y ha brindado asesoría técnica en temas de farmacoeconomía y evaluación de tecnologías sanitarias a distintas compañías farmacéuticas.

El resto de autores declara no tener conflicto de intereses relacionados con el contenido de este documento.

\section{RESPONSABILIDADES ÉTICAS}

Protección de personas y animales. Los autores declaran que para esta investigación no se han
1. IDF Diabetes Atlas. 8th Edition. 2017. Bélgica: International Diabetes Federation; 2017.

2. NCD Risk Factor Collaboration (NCD-RisC). Worldwide trends in diabetes since 1980: a pooled analysis of 751 population-based studies with 4.4 million participants. Lancet. 2016;387(10027):1513-30.

3. Carrillo-Larco RM, Bernabé-Ortiz A. Diabetes mellitus tipo 2 en Perú: una revisión sistemática sobre la prevalencia e incidencia en población general. Rev. Peru. Med. Exp. Salud Pública. 2019;36:26-36.

4. Villena JE. Diabetes mellitus in Peru. Ann Glob Health. 2015;81(6): 765-75.

5. Seclen SN, Rosas ME, Arias AJ, Huayta E, Medina CA. Prevalence of diabetes and impaired fasting glucose in Peru: report from PERUDI$A B$, a national urban population-based longitudinal study. BMJ Open Diabetes Res Care. 2015;3(1):e000110.

6. Tabak AG, Herder C, Rathmann W, Brunner EJ, Kivimaki M. Prediabetes: a high-risk state for diabetes development. Lancet. 2012; 379(9833):2279-90.

7. McPherson S, Reese C, Wendler MC. Methodology update: Delphi studies. Nurs Res. 2018;67(5):404-10.

8. Rosas-Saucedo J, Caballero E, Brito-Córdova G, editores. Consenso de prediabetes. Documento de posición de la Asociación Latinoamericana de Diabetes (ALAD) [Internet]. Asociación Latinoamericana de Diabetes, Consensos ALAD; 2017. Disponible en: http://alad-america latina.org/wp-content/uploads/2016/10/PREDIABETES.pdf

9. Lopez-Jaramillo P, Calderón C, Castillo J, Escobar ID, Melgarejo E, Parra GA. Prediabetes in Colombia: Expert Consensus. Colomb Med (Cali). 2017;48(4):191-203.

10. Fitch K, Bernstein S, Aguilar M, Burnand B, LaCalle J, Lázaro P, et al. The RAND/UCLA Appropriateness Method User's Manual [Internet]. Santa Monica, CA: RAND Corporation; 2001. Disponible en: https:// www.rand.org/pubs/monograph_reports/MR1269.html

11. Martínez-Sahuquillo Amuedo ME, Echevarría Ruiz de Vargas MC. Métodos de consenso. Uso adecuado de la evidencia en la toma de decisiones. «Método RAND/UCLA». Rehabilitación. 2001;35(6):388-92.

12. American Diabetes Association. Classification and diagnosis of diabetes: Standards of medical care in diabetes-2019. Diabetes Care. 2019;42(Suppl 1):S13-28.

13. Hostalek U. Global epidemiology of prediabetes - present and future perspectives. Clin Diabetes Endocrinol. 2019;5:5.

14. López-Jaramillo P, Nieto-Martínez RE, Aure-Fariñez G, Mendivil CO, Lahsen RA, Silava-Filho RL, et al. Identification and management of 
prediabetes: results of the Latin America Strategic Prediabetes Meeting. Rev Panam Salud Publica. 2017;41:e172.

15. Gomez-Arbelaez D, Alvarado-Jurado L, Ayala-Castillo M, Forero-Naranjo L, Camacho PA, Lopez-Jaramillo P. Evaluation of the Finnish Diabetes Risk Score to predict type 2 diabetes mellitus in a Colombian population: A longitudinal observational study. World J Diabetes. 2015;6(17):1337-44.

16. Nieto-Martinez R, Gonzalez-Rivas JP, Aschner P, Barengo NC, Mechanick JI. Transculturalizing diabetes prevention in Latin America. Ann Glob Health. 2017;83(3-4):432-43.

17. Van Name MA, Camp AW, Magenheimer EA, Li F, Dziura JD, Montosa $A$, et al. Effective translation of an intensive lifestyle intervention for hispanic women with prediabetes in a community health center setting. Diabetes Care. 2016;39(4):525-31.

18. Ryden L, Grant PJ, Anker SD, Berne C, Cosentino F, Danchin N, et al. ESC Guidelines on diabetes, pre-diabetes, and cardiovascular diseases developed in collaboration with the EASD: the Task Force on diabetes, pre-diabetes, and cardiovascular diseases of the European Society of Cardiology (ESC) and developed in collaboration with the European Association for the Study of Diabetes (EASD). Eur Heart J. 2013; 34(39):3035-87.
19. Garber AJ, Abrahamson MJ, Barzilay JI, Blonde L, Bloomgarden ZT, Bush MA, et al. Consensus statement by the American Association of Clinical Endocrinologists and American College of Endocrinology on the comprehensive type 2 diabetes management algorithm - 2019 executive summary. Endocr Pract. 2019;25(1):69-100.

20. Consenso peruano sobre prevención y tratamiento de diabetes mellitus tipo 2, síndrome metabólico y diabetes gestacional [Internet]. Perú: Sociedad Peruana de Endocrinología, Sociedad Peruana de Medicina Interna, Congreso Internacional en prediabetes y síndrome metabólico; 25 de agosto de 2012. Disponible en: http://www.endo crinoperu.org/sites/default/files/Consenso\%20Peruano\%20sobre\%20 Prevencion $\% 20 y \% 20$ Tratamiento $\% 20$ de $\% 20$ Diabetes $\% 20$ Mellitus $\% 20$ 2\%20Sindrome\%20Metabolico\%20y\%20Diabetes\%20Gestacional.pdf

21. Lazo-Porras M, Bernabe-Ortiz A, Ruiz-Alejos A, Smeeth L, Gilman RH, Checkley W, et al. Regression from prediabetes to normal glucose levels is more frequent than progression towards diabetes: The CRONICAS Cohort Study. Diabetes Res Clin Pract. 2019:107829.

22. Lopez-Jaramillo P, Gomez-Arbelaez D, Sotomayor-Rubio A, Mantilla-Garcia D, Lopez-Lopez J. Maternal undernutrition and cardiometabolic disease: a Latin American perspective. BMC Med. 2015;13:41. 
ANEXO 1. Filiaciones y cargos de los participantes en el consenso

\begin{tabular}{|c|c|}
\hline Nombre de Experto & Filiación \\
\hline $\begin{array}{l}\text { Dr. Segundo Nicolás } \\
\text { Seclén Santiesteban }\end{array}$ & $\begin{array}{l}\text { Doctor y Maestro en Medicina. Especialista en Endocrinología } \\
\text { Profesor Principal de Medicina y Jefe de la Unidad de Diabetes, Hipertensión y Lípidos, Universidad Peruana } \\
\text { Cayetano Heredia } \\
\text { Past Presidente Sociedad Peruana de Endocrinología }\end{array}$ \\
\hline Dr. Jaime E. Villena Chávez & $\begin{array}{l}\text { Doctor en Medicina, Especialista en Endocrinología del Hospital Nacional Cayetano Heredia } \\
\text { Profesor Principal de Medicina. Universidad Peruana Cayetano Heredia } \\
\text { Académico de Número. Academia Nacional de Medicina } \\
\text { Fellow of American College of Endocrinology } \\
\text { Past-Presidente Sociedad Peruana de Endocrinologia } \\
\text { Miembro Endocrine Society, AACE, ALAD,. }\end{array}$ \\
\hline Dr. Miguel Pinto Valdivia & $\begin{array}{l}\text { Especialista en Endocrinología del Hospital Cayetano Heredia } \\
\text { Clínica Delgado, Presidente ADIPER, Secretario SPE } \\
\text { Membresías del Colegio Americano de Endocrinología (FACE), AACE, ISPAD, ESE, EASD, ENDO SOCIETY }\end{array}$ \\
\hline Dr. José Luis Paz-Ibarra & $\begin{array}{l}\text { Médico Endocrinólogo Hospital Nacional Edgardo Rebagliati Martins. } \\
\text { Profesor Asociado de la Universidad Nacional Mayor de San Marcos. } \\
\text { Miembro titular de la Asociación Americana de Endocrinólogos Clínicos (AACE). } \\
\text { Miembro titular de la Asociación Latinoamérica de Diabetes (ALAD). } \\
\text { Miembro titular de la Sociedad Peruana de Endocrinología (SPE). }\end{array}$ \\
\hline Dr. Ray Willy Ticse Aguirre & $\begin{array}{l}\text { Magister en Epidemiología Clínica (UPCH), Médico especialista en Medicina Interna y Endocrinología (UPCH) } \\
\text { Profesor asociado de la UPCH. Endocrinólogo en el Hospital Cayetano Heredia y Clínica Ricardo Palma } \\
\text { Miembro del consejo directivo de la Asociación de Diabetes del Perú (ADIPER), miembro asociado de la Sociedad } \\
\text { Peruana de Endocrinología. }\end{array}$ \\
\hline Dr. Jesús Rocca Nación & $\begin{array}{l}\text { Endocrinólogo formado en el Hospital Nacional Dos de Mayo (UNMSM) } \\
\text { Estudios de Maestría en Educación en la Universidad Enrique Guzmán y Valle } \\
\text { Endocrinólogo Asociado de la Clínica Ricardo Palma } \\
\text { Ex-endocrinólogo del Hospital Nacional Dos de Mayo } \\
\text { Miembro titular de la Sociedad Peruana de Endocrinología } \\
\text { Miembro Asociado de la Asociación Peruana de Diabetes } \\
\text { Editor y Coautor del Libro «Resistencia a la Insulina» }\end{array}$ \\
\hline $\begin{array}{l}\text { Dr. Harold Lizardo } \\
\text { Torres Aparcana }\end{array}$ & $\begin{array}{l}\text { Médico endocrinólogo (UNMSM). Magíster en Epidemiología (UNMSM). } \\
\text { Miembro del staff de la Clínica San Felipe. } \\
\text { Docente en la Universidad San Martin de Porres. } \\
\text { Miembro de la Sociedad Peruana de Endocrinología, de la Asociación de Diabetes del Perú (ADIPER), de la } \\
\text { Asociación Peruana de Estudio de la Obesidad y Ateroesclerosis (APOA) y de la American Association of Clinical } \\
\text { Endocrinologists (AACE). }\end{array}$ \\
\hline $\begin{array}{l}\text { Dr. Juan Carlos Lizarzarburu } \\
\text { Robles }\end{array}$ & $\begin{array}{l}\text { Medico especialista en endocrinología y maestría en administración de servicios de salud. } \\
\text { Hospital Central de la Fuerza Aérea del Perú, profesor de la Facultad de Medicina de la Universidad Científica del } \\
\text { Sur, profesor de la Facultad de Medicina de Universidad de San Martín de Porres. } \\
\text { Presidente de la asociación para la prevención, educación en investigación en diabetes- APREDIAB. Presidente } \\
\text { electo del capítulo peruano de American Association of Clinical Endocrinologist- AACE }\end{array}$ \\
\hline $\begin{array}{l}\text { Dr. Alberto Alejandro } \\
\text { Teruya Gibu }\end{array}$ & $\begin{array}{l}\text { Endocrinólogo. Hospital Nacional Edgardo Rebagliati Martins. Clínica Centenario Peruano Japonesa, Policlínico } \\
\text { Peruano Japonés. } \\
\text { Profesor tiempo parcial de la Facultad de Medicina de San Fernando de la Universidad Nacional Mayor de San } \\
\text { Marcos y de la Universidad Alas Peruanas. } \\
\text { Miembro de la Sociedad Peruana de Endocrinología; Miembro y Ex Junta Directiva de la Asociación de diabetes } \\
\text { del Perú; Ex miembro del Comité Nacional del Programa de Reforma de Vida en el Seguro Social. }\end{array}$ \\
\hline
\end{tabular}


ANEXO 2. Cuestionario utilizado para el consenso de expertos

\section{DEFINICIÓN PREDIABETES}

Se hizo una búsqueda preliminar de la información disponible en las principales instituciones internacionales. Esta información se presenta a continuación.

\begin{tabular}{|l|l|l|l|}
\hline Fuente/Test & Hemoglobina glicosilada & Glucosa plasmática en ayunas & Test de tolerancia a la glucosa \\
\hline $\begin{array}{l}\text { Asociación Americana de } \\
\text { Diabetes (ADA) (1) }\end{array}$ & $\begin{array}{l}5.7 \% \text { a } 6.4 \% \\
(39-47 \mathrm{mmol}-\mathrm{mol})\end{array}$ & $\begin{array}{l}100 \mathrm{mg} / \mathrm{dl} \mathrm{a} 125 \mathrm{mg} / \mathrm{dl} \\
(5.6-6.9 \mathrm{mmol} / \mathrm{L})\end{array}$ & $\begin{array}{l}140 \mathrm{mg} / \mathrm{dl} \mathrm{a} 199 \mathrm{mg} / \mathrm{dl} \\
(7.8-11.0 \mathrm{mmol} / \mathrm{L})\end{array}$ \\
\hline $\begin{array}{c}\text { Organización mundial de la salud } \\
\text { (OMS) (2) }\end{array}$ & No definido & $\begin{array}{l}110 \mathrm{mg} / \mathrm{dl} \mathrm{a} 125 \mathrm{mg} / \mathrm{dl} \\
(6.1-6.9 \mathrm{mmol} / \mathrm{L})\end{array}$ & No definido \\
\hline
\end{tabular}

1. De acuerdo con su experiencia, ¿los valores propuestos por ADA son extrapolables a la población peruana?

(En una escala del 1 al 9, marque 1 si está totalmente en desacuerdo; marque 9 si está totalmente de acuerdo. El valor 5 indica «ni de acuerdo ni en desacuerdo»)

\begin{tabular}{|l|l|l|l|l|l|l|l|l|}
\hline 1 & 2 & 3 & 4 & 5 & 6 & 7 & 8 & 9 \\
\hline
\end{tabular}

2. De acuerdo con su experiencia, ¿los valores propuestos por OMS son extrapolables a la población peruana?

(En una escala del 1 al 9 marque 1 si está totalmente en desacuerdo y 9 si está totalmente de acuerdo. El valor 5 indica «ni de acuerdo ni en desacuerdo»)

\begin{tabular}{|l|l|l|l|l|l|l|l|l|}
\hline 1 & 2 & 3 & 4 & 5 & 6 & 7 & 8 & 9 \\
\hline
\end{tabular}

3. Usted conoce otra definición de punto de corte para diabetes que sería más precisa para la población peruana?

No $\square$

Si $\square$; por favor que cuestionario y, si es posible, la fuente

\section{TAMIZAJE DE PREDIABETES: CUESTIONARIO}

El cuestionario FINDRISK es una herramienta para la identificación de pacientes con prediabetes. (3) Esta herramienta ha sido validada en diferentes paises incluyendo Colombia (4) y Venezuela (5) para la identificación de pacientes con prediabetes con resultados positivos.

4. ¿Se debería utilizar el cuestinario FINDRISK para la realidad peruana?

(En una escala del 1 al 9 marque 1 si está totalmente en desacuerdo y 9 si está totalmente de acuerdo. El valor 5 indica «ni de acuerdo ni en desacuerdo»)

\begin{tabular}{|l|l|l|l|l|l|l|l|l|}
\hline 1 & 2 & 3 & 4 & 5 & 6 & 7 & 8 & 9 \\
\hline
\end{tabular}

5. ¿Considera necesaria la modificación de esta herramienta para el escenario peruano?

(En una escala del 1 al 9 marque 1 si está totalmente en desacuerdo y 9 si está totalmente de acuerdo. El valor 5 indica «ni de acuerdo ni en desacuerdo»)

\begin{tabular}{|l|l|l|l|l|l|l|l|l|}
\hline 1 & 2 & 3 & 4 & 5 & 6 & 7 & 8 & 9 \\
\hline
\end{tabular}

6. Si su respuesta fue $\geq 6$, ¿qué ítems considera necesario modificar?

7. ¿Considera que se podría utilizar otro cuestionario para la realidad peruana?

No $\square$

Si $\square$; por favor que cuestionario y, si es posible, la fuente

\section{TAMIZAJE Y DIAGNOSTICO DE PREDIABETES: PRUEBAS DE LABORATORIO}

La ADA recomienda que la prueba de glucosa en la sangre para adultos comience a los 45 años, o antes si tiene sobrepeso y tiene factores de riesgo adicionales para la prediabetes o la diabetes tipo 2. Además, debido a que la DM-2 es cada vez más común en niños y adolescentes, probablemente debido al aumento de la obesidad infantil, la ADA recomienda la prueba de prediabetes para los niños con sobrepeso u obesos y que tienen al menos otros dos factores de riesgo para la diabetes tipo 2.

8. ¿Esta de acuerdo con solicitar pruebas de tamizaje de diabetes y prediabetes a los 45 años en sujetos sanos asintomáticos?

(En una escala del 1 al 9 marque 1 si está totalmente en desacuerdo y 9 si está totalmente de acuerdo. El valor 5 indica «ni de acuerdo ni en desacuerdo»)

\begin{tabular}{|l|l|l|l|l|l|l|l|l|}
\hline 1 & 2 & 3 & 4 & 5 & 6 & 7 & 8 & 9 \\
\hline
\end{tabular}

9. Si la respuesta es $\leq 4$, a qué edad se debería solicitar pruebas de tamizaje de diabetes y prediabetes en sujetos asintomáticos?. Si es posible indique la fuente

10. ¿Cuál de las siguientes pruebas considera adecuada para el tamizaje de prediabetes en población peruana?

Glucosa en ayunas:

(En una escala del 1 al 9 marque 1 si está totalmente en desacuerdo y 9 si está totalmente de acuerdo. El valor 5 indica «ni de acuerdo ni en desacuerdo»)

\begin{tabular}{|l|l|l|l|l|l|l|l|l|}
\hline 1 & 2 & 3 & 4 & 5 & 6 & 7 & 8 & 9 \\
\hline
\end{tabular}




\section{Hemoglobina glicosilada:}

(En una escala del 1 al 9 marque 1 si está totalmente en desacuerdo y 9 si está totalmente de acuerdo. El valor 5 indica «ni de acuerdo ni en desacuerdo»)

\begin{tabular}{|l|l|l|l|l|l|l|l|l|}
\hline 1 & 2 & 3 & 4 & 5 & 6 & 7 & 8 & 9 \\
\hline
\end{tabular}

Combinación de estas dos pruebas:

(En una escala del 1 al 9 marque 1 si está totalmente en desacuerdo y 9 si está totalmente de acuerdo. El valor 5 indica «ni de acuerdo ni en desacuerdo»)

\begin{tabular}{|l|l|l|l|l|l|l|l|l|}
\hline 1 & 2 & 3 & 4 & 5 & 6 & 7 & 8 & 9 \\
\hline
\end{tabular}

Otras pruebas de laboratorio, especificar

11. Se debería realizar Test de tolerancia a la glucosa a todos los pacientes con glucosa alterada en ayunas?

(En una escala del 1 al 9 marque 1 si está totalmente en desacuerdo y 9 si está totalmente de acuerdo. El valor 5 indica «ni de acuerdo ni en desacuerdo»)

\begin{tabular}{|l|l|l|l|l|l|l|l|l|}
\hline 1 & 2 & 3 & 4 & 5 & 6 & 7 & 8 & 9 \\
\hline
\end{tabular}

12. Se debería realizar la prueba de HbA1c para diagnóstico de Prediabetes en el Perú?

(En una escala del 1 al 9 marque 1 si está totalmente en desacuerdo y 9 si está totalmente de acuerdo. El valor 5 indica «ni de acuerdo ni en desacuerdo»)

\begin{tabular}{|l|l|l|l|l|l|l|l|l|}
\hline 1 & 2 & 3 & 4 & 5 & 6 & 7 & 8 & 9 \\
\hline
\end{tabular}

\section{EPIDEMIOLOGÍA}

Con respecto a la prevalencia de prediabetes, el estudio PERUDIAB 2012 realizado en 1677 hogares a nivel nacional, representativo de más de 10 millones de adultos mayores de 25 años, encontró una prevalencia de $22.4 \%$ para hiperglicemia de ayuno (prediabetes). (6)

\section{3. ¿Usted está de acuerdo con esta información?}

(En una escala del 1 al 9 marque 1 si está totalmente en desacuerdo y 9 si está totalmente de acuerdo. El valor 5 indica «ni de acuerdo ni en desacuerdo»)

\begin{tabular}{|l|l|l|l|l|l|l|l|l|}
\hline 1 & 2 & 3 & 4 & 5 & 6 & 7 & 8 & 9 \\
\hline
\end{tabular}

¿Esta información sobreestima la realidad nacional?

(En una escala del 1 al 9 marque 1 si está totalmente en desacuerdo y 9 si está totalmente de acuerdo. El valor 5 indica «ni de acuerdo ni en desacuerdo»)

\begin{tabular}{|l|l|l|l|l|l|l|l|l|}
\hline 1 & 2 & 3 & 4 & 5 & 6 & 7 & 8 & 9 \\
\hline
\end{tabular}

¿Esta información subestima la realidad nacional?

(En una escala del 1 al 9 marque 1 si está totalmente en desacuerdo y 9 si está totalmente de acuerdo. El valor 5 indica «ni de acuerdo ni en desacuerdo»)

\begin{tabular}{|l|l|l|l|l|l|l|l|l|}
\hline 1 & 2 & 3 & 4 & 5 & 6 & 7 & 8 & 9 \\
\hline
\end{tabular}

14. Existe alguna otra fuente que estime la prevalencia de prediabetes en el Perú

Además, en el estudio PERUDIAB, se encontró un mayor riesgo de diabetes en población de la costa y sexo masculino. La prevalencia fue mayor en los grupos mayores de 35 años comparados con el grupo de 25 a 34 años. En este estudio no se evaluaron poblaciones menores de 25 años.

Por favor, indicar si usted esta de acuerdo con las siguientes afirmaciones del estudio de acuerdo a su experiencia:

15. En el Perú hay un mayor riesgo de diabetes en población de la costa:

(En una escala del 1 al 9 marque 1 si está totalmente en desacuerdo y 9 si está totalmente de acuerdo. El valor 5 indica «ni de acuerdo ni en desacuerdo»)

\begin{tabular}{|l|l|l|l|l|l|l|l|l|}
\hline 1 & 2 & 3 & 4 & 5 & 6 & 7 & 8 & 9 \\
\hline
\end{tabular}

16. En el Perú hay un mayor riesgo de diabetes en población de sexo masculino:

(En una escala del 1 al 9 marque 1 si está totalmente en desacuerdo y 9 si está totalmente de acuerdo. El valor 5 indica «ni de acuerdo ni en desacuerdo»)

\begin{tabular}{|l|l|l|l|l|l|l|l|l|}
\hline 1 & 2 & 3 & 4 & 5 & 6 & 7 & 8 & 9 \\
\hline
\end{tabular}

17. En el Perú la prevalencia es mayor en los grupos mayores de 35 años comparados con el grupo de 25 a 34 años.

(En una escala del 1 al 9 marque 1 si está totalmente en desacuerdo y 9 si está totalmente de acuerdo. El valor 5 indica «ni de acuerdo ni en desacuerdo»)

\begin{tabular}{|l|l|l|l|l|l|l|l|l|}
\hline 1 & 2 & 3 & 4 & 5 & 6 & 7 & 8 & 9 \\
\hline
\end{tabular}

18. ¿Existe otro grupo de mayor riesgo de diabetes en nuestro país?

\section{INTERVENCIONES DE MANEJO}

Existe evidencia de daño vascular en la fase de Prediabetes, por lo que la rapida identificación y manejo es fundamental. En el Perú, no se ha identificado estudios de evaluación económica en salud que evaluen la costo-efectividad de intervenciones intensivas (farmacológica y/o cambio de estilo de vida) de la Prediabetes en el Perú.

\section{En su opinión, qué estrategia sería costo-efectiva?}

Existen estudios en otros paises, por ejemplo Estados Unidos, que evalúan la costo efectividad de estrategias de manejo y prevención de diabetes. Estos demuestran que cualquiera de las estrategias modificadoras del estilo de vida así como tratamiento farmacológico con metformina serían costo efectivas en ese escenario. (3) 
20. ¿Considera que los cambios intensivos del estilo de vida, como los descritos en el estudio US-DPP, sería una intervención aplicable en la población peruana?

(En una escala del 1 al 9 marque 1 si está totalmente en desacuerdo y 9 si está totalmente de acuerdo. El valor 5 indica «ni de acuerdo ni en desacuerdo»)

\begin{tabular}{|l|l|l|l|l|l|l|l|l|}
1 & 2 & 3 & 4 & 5 & 6 & 7 & 8 & 9 \\
\hline
\end{tabular}

21. Si su respuesta fue $\leq 4$, qué sería necesario modificar para su adecuada implementación en el país?

22. ¿Considera que los cambios del estilo de vida de acuerdo al «mundo real» («real-world effectiveness»), como los descritos en el estudio PREVENT-DM para población latina de USA, sería una intervención aplicable en la población peruana?

(En una escala del 1 al 9 marque 1 si está totalmente en desacuerdo y 9 si está totalmente de acuerdo. El valor 5 indica «ni de acuerdo ni en desacuerdo»)

\begin{tabular}{|l|l|l|l|l|l|l|l|l|}
\hline 1 & 2 & 3 & 4 & 5 & 6 & 7 & 8 & 9 \\
\hline
\end{tabular}

23. Si su respuesta fue $\leq 4$, qué sería necesario modificar para su adecuada implementación en el país?

La metformina es una biguanida que reduce la producción de glucosa en el hígado y causa una pérdida de peso moderada en pacientes obesos y con sobrepeso. (7) Se demostró que el uso de una dosis de 850 mg dos veces al día reduce la progresión de la prediabetes a la diabetes en el 31\% de los participantes frente al grupo de placebo. (7)

24. Con respecto al tratamiento farmacológico con metformina, considera que sería una intervención adecuada en población peruana?

(En una escala del 1 al 9 marque 1 si está totalmente en desacuerdo y 9 si está totalmente de acuerdo. El valor 5 indica «ni de acuerdo ni en desacuerdo»)

\begin{tabular}{|l|l|l|l|l|l|l|l|l|}
\hline 1 & 2 & 3 & 4 & 5 & 6 & 7 & 8 & 9 \\
\hline
\end{tabular}

25. Si su respuesta fue $\geq 6$, ¿Por cuánto tiempo y en qué dosis indicaría metformina para la realidad nacional?

26. Qué criterios son necesarios para evaluar la respuesta a estas intervenciones?

27. Qué tan frecuente debe ser el seguimiento del paciente prediabético?
28. Con respecto al tratamiento farmacológico con metformina, considera que debería ser una intervención de segunda línea respecto a la intervención inicial de cambios del estilo de vida según el «mundo real»?

(En una escala del 1 al 9 marque 1 si está totalmente en desacuerdo y 9 si está totalmente de acuerdo. El valor 5 indica «ni de acuerdo ni en desacuerdo»)

\begin{tabular}{|l|l|l|l|l|l|l|l|l|}
\hline 1 & 2 & 3 & 4 & 5 & 6 & 7 & 8 & 9 \\
\hline
\end{tabular}

29. En qué casos considera que la metformina debería ser una intervención concomitante a la intervención de cambios del estilo de vida según el «mundo real»?

30. ¿Considera que existe sinergia entre la administración de metformina y los cambios en el estilo de vida en el «mundo real» para reducir el progreso a diabetes?

(En una escala del 1 al 9 marque 1 si está totalmente en desacuerdo y 9 si está totalmente de acuerdo. El valor 5 indica «ni de acuerdo ni en desacuerdo»)

\begin{tabular}{|l|l|l|l|l|l|l|l|l|}
\hline 1 & 2 & 3 & 4 & 5 & 6 & 7 & 8 & 9 \\
\hline
\end{tabular}

\section{REFERENCIAS}

1. Classification and Diagnosis of Diabetes: Standards of Medical Care in Diabetes-2019. Diabetes Care. 2019;42(Suppl 1):S13-s28.

2. Hostalek U. Global epidemiology of prediabetes - present and future perspectives. Clin Diabetes Endocrinol. 2019;5:5.

3. López-Jaramillo P, Nieto-Martínez RE, Aure-Fariñez G, Mendivil CO, Lahsen RA, RL S-F. Identification and management of prediabetes: results of the Latin America Strategic Prediabetes Meeting. Rev Panam Salud Publica. 2017;41.

4. Gomez-Arbelaez D, Alvarado-Jurado L, Ayala-Castillo M, Forero-Naranjo L, Camacho PA, Lopez-Jaramillo P. Evaluation of the Finnish Diabetes Risk Score to predict type 2 diabetes mellitus in a Colombian population: A longitudinal observational study. World J Diabetes. 2015;6(17):1337-44.

5. Nieto-Martinez R, Gonzalez-Rivas JP, Aschner P, Barengo NC, Mechanick Jl. Transculturalizing Diabetes Prevention in Latin America. Ann Glob Health. 2017;83(3-4):432-43.

6. Seclen SN, Rosas ME, Arias AJ, Huayta E, Medina CA. Prevalence of diabetes and impaired fasting glucose in Peru: report from PERUDI$A B$, a national urban population-based longitudinal study. BMJ Open Diabetes Res Care. 2015;3(1):e000110.

7. Ryden L, Grant PJ, Anker SD, Berne C, Cosentino F, Danchin N, et al. ESC Guidelines on diabetes, pre-diabetes, and cardiovascular diseases developed in collaboration with the EASD: the Task Force on diabetes, pre-diabetes, and cardiovascular diseases of the European Society of Cardiology (ESC) and developed in collaboration with the European Association for the Study of Diabetes (EASD). Eur Heart J. 2013;34(39):3035-87. 